\title{
Patterned Nanofoam Fabrication from a Variety of Materials via Femtosecond Laser Pulses
}

\author{
James A. Grant-Jacob*, Benita S. Mackay, James A. G. Baker, Yunhui Xie, Michael D. T. McDonnell, \\ Daniel J. Health, Matthew Praeger, Robert W. Eason, Ben Mills
}

Optoelectronics Research Centre, University of Southampton, Southampton, UK

Email: ${ }^{* j a g j 1 v 11 @ s o t o n . a c . u k}$

How to cite this paper: Grant-Jacob, J.A., Mackay, B.S., Baker, J.A.G., Xie, Y.H., McDonnell, M.D.T., Health, D.J., Praeger, M., Eason, R.W. and Mills, B. (2019) Patterned Nanofoam Fabrication from a Variety of Materials via Femtosecond Laser Pulses. Materials Sciences and Applications, 10, 186-196.

https://doi.org/10.4236/msa.2019.103015

Received: December 29, 2018

Accepted: March 9, 2019

Published: March 12, 2019

Copyright $\odot 2019$ by author(s) and Scientific Research Publishing Inc. This work is licensed under the Creative Commons Attribution International License (CC BY 4.0).

http://creativecommons.org/licenses/by/4.0/

\begin{abstract}
High-repetition-rate femtosecond lasers enable the precise production of nanofoam from a wide range of materials. Here, the laser-based fabrication of nanofoam from silicon, borosilicate glass, soda-lime glass, gallium lanthanum sulphide and lithium niobate is demonstrated, where the pore size of the nanofoam is shown to depend strongly on the material used, such that the pore width and nanofibre width appear to increase with density and thermal expansion coefficient of the material. In addition, the patterning of nanofoam on a glass slide, with fabricated pattern pixel resolution of $\sim 35 \mu \mathrm{m}$, is demonstrated.
\end{abstract}

\section{Keywords}

Laser Ablation, Nanofibres, Nanofoam, QR Code, Patterning, Lithium Niobate, Gallium Lanthanum Sulphide, Silicon, Silica

\section{Introduction}

A class of porous nanostructured materials, known as nanofoams [1] [2] [3], can be made via a variety of methods, such as laser ablation [4] [5] [6]. Laser-based nanofoam fabrication offers the potential for control over the precise location at which the nanofoam is generated. Such fabrication generally involves using a laser operating at a high repetition rate (usually at least $\sim \mathrm{kHz}$ ) delivering femtosecond pulses onto the surface of a material [7]. Upon incidence on the material, the laser pulses can undergo nonlinear absorption, leading to the generation of a plasma via multiphoton ionization and subsequent avalanche ionization [4] [8] [9] [10]. The high number of ultra-short pulses incident on one area of the material, in a short enough time, can lead to the build-up of thermal energy causing the surrounding material to melt [11]-[16]. During this process, molten droplets 
and jets of materials are ejected [4] [14], which, due to their small size, cool rapidly and solidify to form nanofibres that can intertwine to form nanofoam [17]. The volume of the nanofoam, the pore size, and nanofibre width, can depend on fabrication parameters such as the speed of translation of the sample beneath the incident laser beam and the focal conditions of the laser [18].

Such fibrous nanostructures have received great interest recently, owing to their optical and mechanical properties. Potential applications include subwavelength-diameter glass wires for microscale photonic devices [19] and evanescent nanosensors [20], nanoscale fibres for advancing silicon photonics [21], and polymer nanofibres for biomedical applications such as tissue templates [22].

Previous work in this research area includes conductivity measurements of carbon nanofoam [7], $8 \mathrm{MHz}$ fabrication from individual regions in silica [23], the dependence of nanofoam fabrication on depth in glass [18], the structure of $\mathrm{As}_{2} \mathrm{~S}_{3}$ nanofibers [5], and a discussion of the likely mechanism for nanofoam fabrication [4]. Here, for the first time, by comparingnanofoam from a range of materials, we show how the fabricated nanofoam depends on several of the material parameters, and we also demonstrate a method for the patterning of the fabricated nanofoam.

Here, we use $\sim 150$ fs duration laser pulses to fabricate nanofoam from silicon (a common material used in electronics and mid-IR photonics) [6] [24], silica-based glass (used in a wide range of photonics applications) [25], gallium lanthanum sulphide, also known as GLS (a material used in active and passive infrared applications) [26], and lithium niobate (a ferroelectric material used in nonlinear optical applications) [27] [28]. We also demonstrate the ability to fabricate patterned regions of nanofoam on a borosilicate glassslide.

\section{Experimental Setup}

The laser-based fabrication of nanofoam generally requires the raster-scanning of a focussed laser spot across the material surface. The laser pulse energy, laser focus spot size, the spacing between adjacent raster-scanned lines and the raster-scan line speed are all parameters that can be optimized for the fabrication process. Figure 1 shows a schematic of the experimental setup used for nanofoam fabrication, while Table 1 contains the key laser parameters used in the experiment.

Ultrashort pulses of $\sim 150$ fs duration and central wavelength of $800 \mathrm{~nm}$, from an ultrafast Ti: sapphire laser system operating at a repetition rate of $250 \mathrm{kHz}$, delivering an energy of $\sim 4 \mu \mathrm{J}$ per pulse, were used to fabricate the nanofoam. The laser pulses passed through a computer-controlled shutter and a $4 \mathrm{~mm}$ diameter circular aperture, to convert a Gaussian spatial intensity into one that closely resembled a top-hat spatial intensity. Subsequently, the pulses were demagnified using a 50× microscope objective lens (Nikon LU Plan, NA $=0.55$ ) and imaged to a beam diameter of $\sim 4 \mu \mathrm{m}$ on the surface of a material, which resulted in a laser pulse energy density of $\sim 15 \mathrm{~J} \cdot \mathrm{cm}^{-2}$ at the surface of the material. 


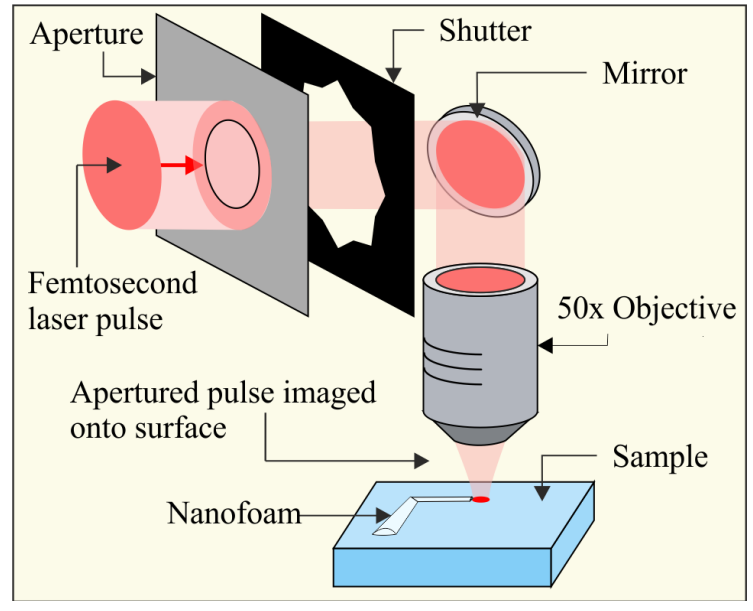

Figure 1. Schematic of the experimental setup for fabrication of nanofoam on the surface of a material.

Table 1. Table displaying the key laser parameters.

\begin{tabular}{cc}
\hline Repetition Rate $(\mathrm{kHz})$ & 250 \\
Pulse width $(\mathrm{fs})$ & $\sim 150$ \\
Central wavelength $(\mathrm{nm})$ & 800 \\
Beam spot size at surface $(\mu \mathrm{m})$ & $\sim 4$ \\
Laser fluence $\left(\mathrm{J} \cdot \mathrm{cm}^{-2}\right)$ & 15 \\
Scanning speed $\left(\mathrm{mm} \cdot \mathrm{s}^{-1}\right)$ & 1 \\
Raster-scan line separation $(\mu \mathrm{m})$ & 5 \\
\hline
\end{tabular}

The materials were mounted on a 3-axis stage that was computer controlled to allow automated raster-scan translation of the material, hence effectively scanning the laser focus along the surface of the material. This enabled the fabrication of individual lines of nanofoam to form a contiguous region of nanofoam, as described in previous work [18].

For consistency, we used a raster-scan line speed of $1 \mathrm{~mm} \cdot \mathrm{s}^{-1}$ and raster-scan line separation of $5 \mu \mathrm{m}$ for all materials, where the lateral dimensions of the fabricated nanofoam was on the order of a few hundred microns. Five different materials, namely silicon, borosilicate glass, sodalime glass, GLS and lithium niobate were investigated.

\section{Results and Discussion}

In this experimental section, results from each of the five materials are presented in Sections 3.1 to 3.5. In each case, a low-resolution and high-resolution scanning electron microscope (SEM) image is presented, along with analysis of the nanofoam parameters. Here, the pore size and the nanofibre width were calculated directly from the high-resolution images by using image analysis software to measure the mean width over 10 positions. A comparison is presented in Section 3.6, and results for high-precision patterning are shown in Section 3.7. Laser 
and raster-scan parameters were constant for all materials, and hence a direct comparison can be made.

\subsection{Silicon}

The nanofoam fabricated from the polished surface of a p-type (100) silicon wafer is highlighted in the SEM image shown in Figure 2(a). Fragments of coalesced material and nanofibers are visible up to a few hundred microns away from the nanofoam block, demonstrating how far the ablated material can be ejected. Although from the image in Figure 2(a), the nanofoam block appears to be anamalgamation of material, as seen from a highermagnification image of the nanofoam (Figure 2(b)), the width of the fibres that make up the nanofoam are $\sim 90 \mathrm{~nm}$ wide and the average pore size is $\sim 1.1$ micron in width.

\subsection{Borosilicate Glass}

Under the same conditions as the fabrication of silicon, a square region of borosilicate nanofoam was fabricated from a borosilicate glass slide (BK7, $75 \times 25 \times$ $0.7 \mathrm{~mm}$ ). An SEM image of the nanofoam region is presented in Figure 3(a). In this case there is less visible ejection of material outside of the ablated region, and, in particular, far fewer filamentary structures are present. The ejected material instead takes the form of very small particles with occasional larger clumps. Determined from the higher magnification image in Figure 3(b), an average nanofibrewidth of $\sim 120 \mathrm{~nm}$ was calculated, and an average pore width of $\sim 1.2$ microns was calculated.

\subsection{Soda Lime Glass}

To provide a comparison for a different type of silica glass, nanofoam was fabricated from soda lime glass, which in this work was in the form of a coverslip $(24 \times 50 \times 0.16 \mathrm{~mm})$. The nanofoam produced was visually different from those produced from the borosilicate glass. From Figure 4(a), the average pore size of

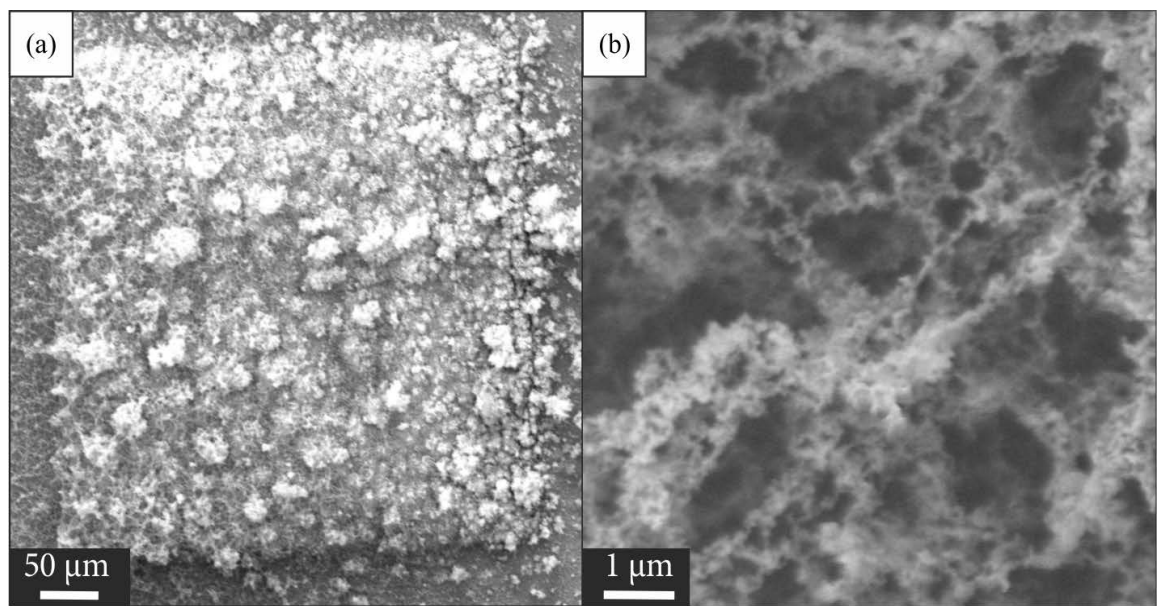

Figure 2. SEM images of the silicon nanofoam showing (a) the $0.45 \mathrm{~mm}$ by $0.45 \mathrm{~mm}$ total area and (b) higher magnification detail of the nanofoam fibres. 


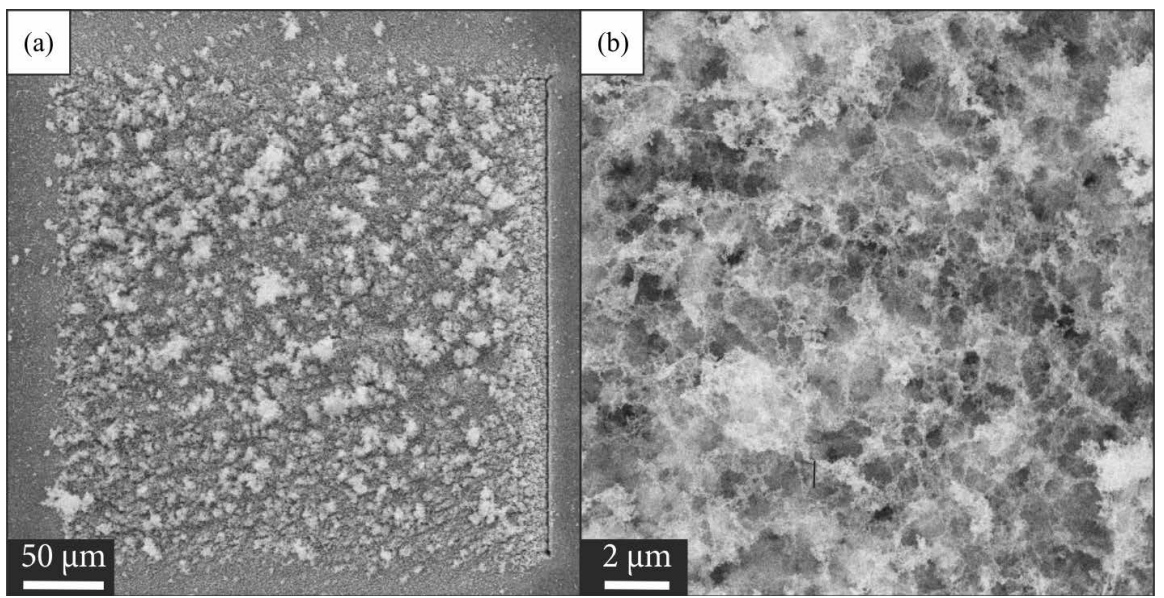

Figure 3. SEM images of the borosilicate nanofoam showing (a) the $0.3 \mathrm{~mm}$ by $0.3 \mathrm{~mm}$ total area and (b) higher magnification detail of the nanofoam fibres.

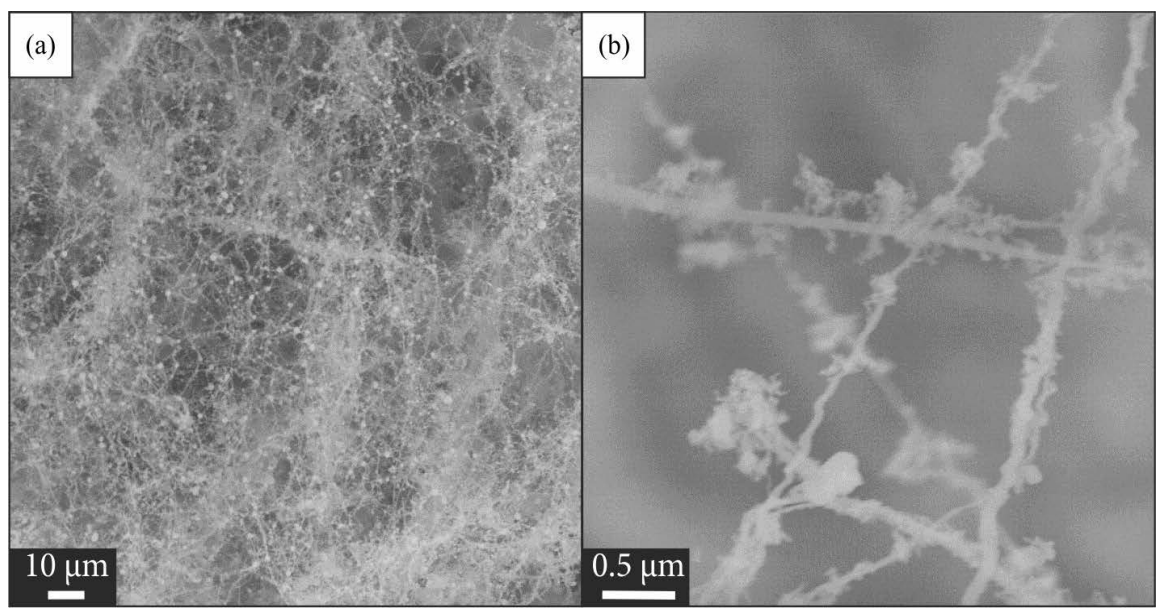

Figure 4. SEM images of the soda-lime nanofoam showing (a) the presence of beads of material at the end of the nanofibers, and (b) higher magnification detail for single nanofibers.

$\sim 2.3 \mu \mathrm{m}$ and nanofiber width of $\sim 300 \mathrm{~nm}$ is shown.

\subsection{Gallium Lanthanum Sulphide}

From the SEM image shown in Figure 5(a), it is evident that the nanofoam structure produced average pore size of $\sim 2.3 \mu \mathrm{m}$ and nanofiber width of $\sim 300$ $\mathrm{nm}$. Using a scalpel to dig into the nanofoam volume and drag the nanofoam, it was also possible to stretch the nanofoam, which then remained in its deformed position as shown in Figure 5(b).

\subsection{Lithium Niobate}

Compared to the other materials the nanofoam produced via the ablation of lithium niobate was produced away from the ablation area, and not in the location of laser irradiation, as shown in Figure 6(a). The nanofoam appears to have been generated up to $\sim 1 \mathrm{~mm}$ away from the laser irradiatedregion. There also 


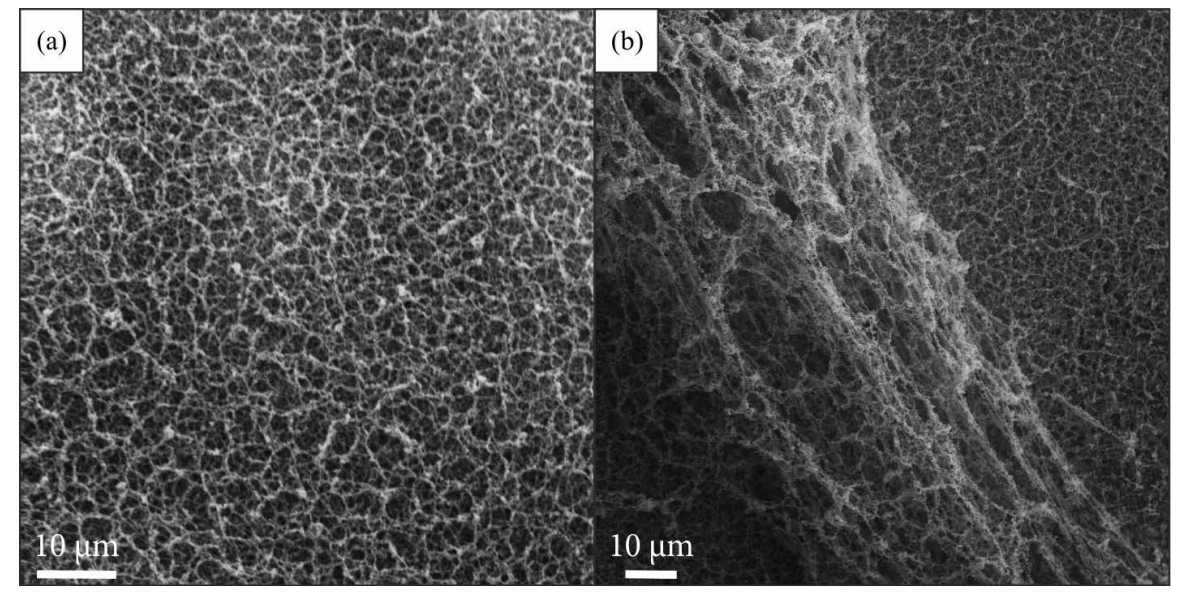

Figure 5. High resolution SEM images of the GLS nanofoam showing (a) nanofoam detail and (b) the ability to mechanically deform the nanofoam.

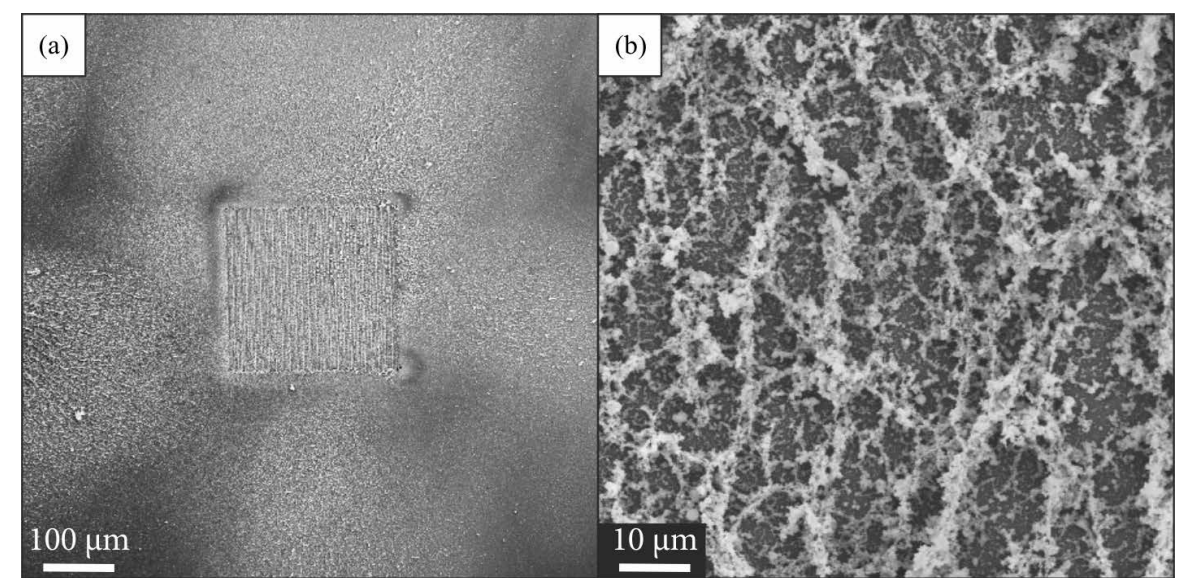

Figure 6. SEM images of the lithium niobate nanofoam showing (a) the $0.25 \mathrm{~mm}$ by 0.25 $\mathrm{mm}$ total area and (b) higher magnification detail of the nanofoam fibres, outside the laser irradiated region, where in general the nanofibers are orientated in the preferential direction.

appears to be a directionality to the nanofibres of the foam. The average nanofibre width was $\sim 750 \mathrm{~nm}$ and the average pore width was $\sim 7 \mu \mathrm{m}$.

\subsection{Comparison for the Materials}

Table 2 shows a comparison between the nanofoam materials fabricated, for the average pore width and the average nanofiber width, as measured from the SEM images in Sections 3.1 to 3.5. The results indicate a correlation between average pore width and nanofibre width.

This correlation can be seen clearly from the plot in Figure 7(a), which shows average pore width versus average nanofibre width. Plotted in Figure $7(\mathrm{~b})$ are the average pore width and average nanofibre width as a function of bulk material density. In this plot the trend shows that increasing density increases average pore width and nanofibre width. A similar increasing trend in average pore width and nanofibre width is also observed with increasing thermal expansion 
Table 2. Table displaying the approximate avarage pore width and approximate nanofibre width of the nanofoams produced from the different materials.

\begin{tabular}{ccc}
\hline Material & Average pore width $(\mu \mathrm{m})$ & Average nanofibre width $(\mathrm{nm})$ \\
\hline Silicon & 1.1 & 90 \\
Borosilicate Glass & 1.2 & 120 \\
Soda Lime Glass & 4.88 & 340 \\
GLS & 2.3 & 300 \\
Lithium Niobate & 7 & 750 \\
\hline
\end{tabular}
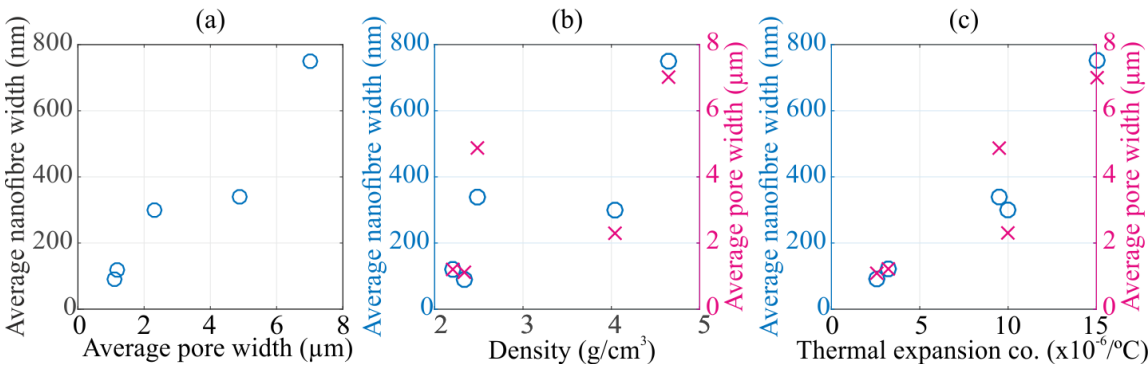

Figure 7. (a) Average pore width as a function of nanofibre width from the nanofoams produced in this work. Average pore width and average nanofibre width as a function of (b) density of the bulk material and (c) thermal expansion coefficient of the material. Values of density and thermal expansion coefficient at $25^{\circ} \mathrm{C}$ obtained from [29] [30] [31] [32].

coefficient, as shown Figure 7(c). These trends are perhaps understandable owing to less dense material being more easily ablated with material having more energy and travelling further from the material, thus creating larger pores. Likewise, ejected melted material that expands faster due to a higher thermal expansion would likely lead to larger pores.

\subsection{Nanofoam Patterning}

In order to demonstrate the versatility of the fabrication method and potential applications, we chose to produce patterned areas of nanofoam. For this demonstration, the computer-controlled shutter was automatically opened and closed, whilst the laser focus was continually raster-scanned over the surface of the material, in order to laser irradiate the intended pattern. The size of the patterns was chosen as a balance between minimum time of fabrication and maximum possible resolution for potential imaging using a smart phone camera, for example. The patterns chosen for the nanofoam fabrication were a wheel and a QR code, where the designs were implemented by the automated stages via Matlab and LabVIEW. Both patterns were fabricated on a borosilicate glass slide, each over $2 \mathrm{~mm} \times 2 \mathrm{~mm}$ square area. For proof of principle and ease of machining in straight lines, the patterns were chosen to be formed of square pixels. Each pixel on the 32 pixel $\times 32$ pixel wheel image corresponded to a $65 \mu \mathrm{m} \times 65 \mu \mathrm{m}$ machined area, and each pixel on the 16 pixel $\times 16$ pixel $Q R$ code corresponded to a patterned pixel of $35 \mu \mathrm{m} \times 35 \mu \mathrm{m}$ machined area.

An SEM image of the wheel pattern is shown in Figure 8(a), where the white 


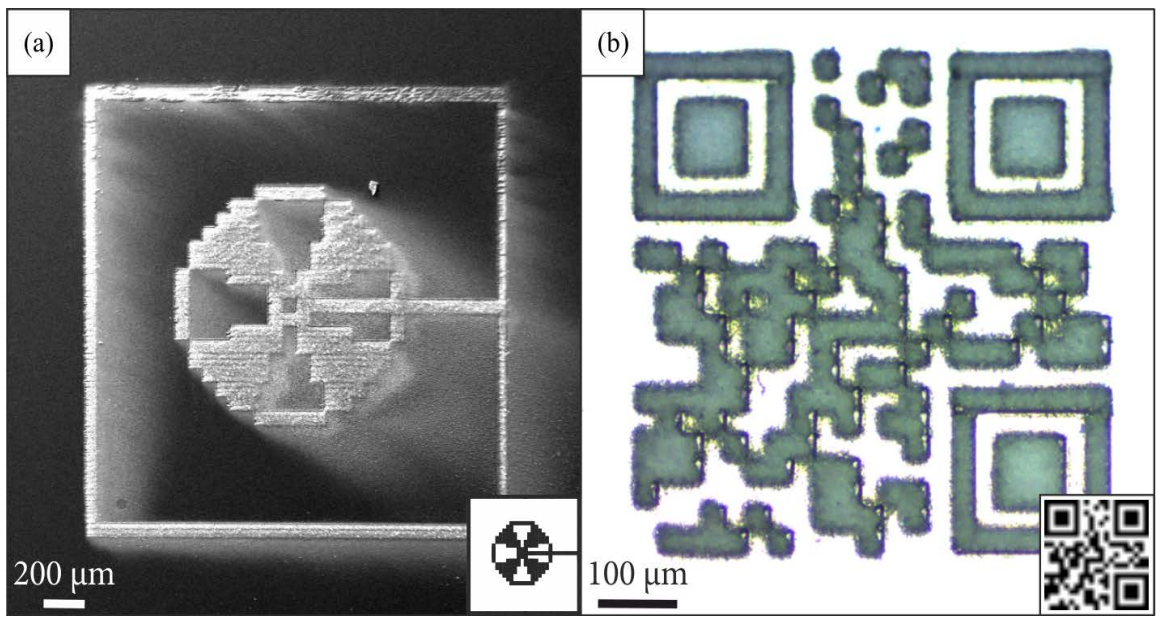

Figure 8. Demonstration of patterned nanofoam, showing (a) an SEM image of a wheel pattern and (b) a microscope image of a QR code.

parts of the image correspond to the regions of nanofoam. The white smearing in the image is a result of imaging nanofoam formed from ablated material that has been carried in the air that flows in a specific direction over the sample, due to the air conditioning in the laboratory. An optical microscope image of the nanofoam QR code is shown in Figure 8(b), where regions of nanofoam appear as grey, and the non-ablated glass as white. Inset to both figures is the monochrome bitmap pattern loaded into the Matlab and LabVIEW program.

\section{Conclusion}

In conclusion, we have demonstrated that femtosecond ablation can be used to fabricate nanofoam from a range of materials, where the porosity of the nanofoam and the width of the wires were shown to be dependent on the particular material. More specifically, the pore width and nanofibre width appeared to increase with density and thermal expansion coefficient of the material. Upon demonstration of the fabrication of patterned nanofoam, including a QR code of glass nanofoam, we found that we were able to successfully fabricate patterns of several hundred microns wide with a machined pattern pixel area of size $35 \mu \mathrm{m} \times$ $35 \mu \mathrm{m}$. Further work will focus on the optimisation of the size and structure of the nanofoam via machine learning [33].

\section{Acknowledgements}

The authors are grateful to the Engineering and Physical Sciences Research Council (EPRSC) under grant No. EP/N03368X/1.The RDM data for this paper can be found at https://doi.org/10.5258/SOTON/D0753.

\section{Conflicts of Interest}

The authors declare no conflicts of interest regarding the publication of this paper. 


\section{References}

[1] Bag, S., Trikalitis, P.N., Chupas, P.J., Armatas, G.S. and Kanatzidis, M.G. (2007) Porous Semiconducting Gels and Aerogels from Chalcogenide Clusters. Science, 317, 490-493. https://doi.org/10.1126/science.1142535

[2] Sun, H., Xu, Z. and Gao, C. (2013) Multifunctional, Ultra-Flyweight, Synergistically Assembled Carbon Aerogels. Advanced Materials, 25, 2554-2560. https://doi.org/10.1002/adma.201204576

[3] Brock, S.L. (2007) Materials Science. Filling a Void. Science, 317, 460-461. https://doi.org/10.1126/science.1146517

[4] Venkatakrishnan, K., Vipparty, D. and Tan, B. (2011) Nanofibre Fabrication by Femtosecond Laser Ablation of Silica Glass. Optics Express, 19, 15770-15776. https://doi.org/10.1364/OE.19.015770

[5] Juodkazis, S., Misawa, H., Louchev, O.A. and Kitamura, K. (2006) Femtosecond Laser Ablation of Chalcogenide Glass: Explosive Formation of Nano-Fibres against Thermo-Capillary Growth of Micro-Spheres. Nanotechnology, 17, 4802-4805. https://doi.org/10.1088/0957-4484/17/19/003

[6] Zhang, Y.F., Tang, Y.H., Wang, N., Yu, D.P., Lee, C.S., Bello, I. and Lee, S.T. (1998) Silicon Nanowires Prepared by Laser Ablation at High Temperature. Applied Physics Letters, 72, 1835. https://doi.org/10.1063/1.121199

[7] Rode, A.V., Gamaly, E.G. and Luther-Davies, B. (2000) Formation of Cluster-Assembled Carbon Nano-Foam by High-Repetition-Rate Laser Ablation. Applied Physics A: Materials Science \& Processing, 70, 135-144. https://doi.org/10.1007/s003390050025

[8] Feit, M.D., Komashko, A.M. and Rubenchik, A.M. (2004) Ultra-Short Pulse Laser Interaction with Transparent Dielectrics. Applied Physics A, 79, 1657-1661. https://doi.org/10.1007/s00339-004-2683-1

[9] Kaiser, A., Rethfeld, B., Vicanek, M. and Simon, G. (2000) Microscopic Processes in Dielectrics under Irradiation by Subpicosecond Laser Pulses. Physical Review B, 61, 11437-11450. https://doi.org/10.1103/PhysRevB.61.11437

[10] Brodeur, A. and Chin, S. (1998) Band-Gap Dependence of the Ultrafast White-Light Continuum. Physical Review Letters, 80, 4406-4409. https://doi.org/10.1103/PhysRevLett.80.4406

[11] Kasaai, M.R., Kacham, V., Theberge, F. and Chin, S.L. (2003) The Interaction of Femtosecond and Nanosecond Laser Pulses with the Surface of Glass. Journal of Non-Crystalline Solids, 319, 129-135. https://doi.org/10.1016/S0022-3093(02)01909-9

[12] Tamaki, T., Watanabe, W. and Itoh, K. (2006) Laser Micro-Welding of Transparent Materials by a Localized Heat Accumulation Effect Using a Femtosecond Fiber Laser at $1558 \mathrm{~nm}$. Optics Express, 14, 10460-10468. https://doi.org/10.1364/OE.14.010460

[13] Koubassov, V., Laprise, J.F., Théberge, F., Chin, S.L., Förster, E., Sauerbrey, R., Müller, B. and Glatzel, U. (2004) Ultrafast Laser-Induced Melting of Glass. Applied Physics A: Materials Science \& Processing, 79, 499-505. https://doi.org/10.1007/s00339-003-2474-0

[14] Tokarev, V.N., Lazare, S., Belin, C. and Debarre, D. (2004) Viscous Flow and Ablation Pressure Phenomena in Nanosecond UV Laser Irradiation of Polymers. Applied Physics A, 79, 717-720. https://doi.org/10.1007/s00339-004-2693-Z

[15] Korte, F., Koch, J. and Chichkov, B.N. (2004) Formation of Microbumps and Na- 
nojets on Gold Targets by Femtosecond Laser Pulses. Applied Physics A, 79, 879-881. https://doi.org/10.1007/s00339-004-2590-5

[16] Ben-Yakar, A., Byer, R.L., Harkin, A., Ashmore, J., Stone, H.A., Shen, M. and Mazur, E. (2003) Morphology of Femtosecond-Laser-Ablated Borosilicate Glass Surfaces. Applied Physics Letters, 83, 3030-3032. https://doi.org/10.1063/1.1619560

[17] Courtier, A.F., Grant-Jacob, J.A., Ismaeel, R., Heath, D.J., Brambilla, G., Stewart, W.J., Eason, R.W. and Mills, B. (2017) Laser-Based Fabrication of Nanofoam inside a Hollow Capillary. Materials Sciences and Applications, 8, 829-837.

[18] Grant-Jacob, J.A., Mills, B. and Eason, R.W. (2014) Parametric Study of the Rapid Fabrication of Glass Nanofoam via Femtosecond Laser Irradiation. Journal of Physics D: Applied Physics, 47, Article ID: 055105.

https://doi.org/10.1088/0022-3727/47/5/055105

[19] Tong, L., Gattass, R.R., Ashcom, J.B., He, S., Lou, J., Shen, M., Maxwell, I. and Mazur, E. (2003) Subwavelength-Diameter Silica Wires for Low-Loss Optical Wave Guiding. Nature, 426, 816-819. https://doi.org/10.1038/nature02193

[20] Brambilla, G. (2010) Optical Fibre Nanowires and Microwires: A Review. Journal of Optics, 12, Article ID: 043001. https://doi.org/10.1088/2040-8978/12/4/043001

[21] Liu, Z.Q., Zhou, W.Y., Sun, L.F., Tang, D.S., Zou, X.P., Li, Y.B., Wang, C.Y., Wang, G. and Xie, S.S. (2001) Growth of Amorphous Silicon Nanowires. Chemical Physics Letters, 341, 523-528. https://doi.org/10.1016/S0009-2614(01)00513-9

[22] Huang, Z.-M., Zhang, Y.-Z., Kotaki, M. and Ramakrishna, S. (2003) A Review on Polymer Nanofibers by Electrospinning and Their Applications in Nanocomposites. Composites Science and Technology, 63, 2223-2253. https://doi.org/10.1016/S0266-3538(03)00178-7

[23] Sivakumar, M., Venkatakrishnan, K. and Tan, B. (2009) Synthesis of Glass Nanofibers Using Femtosecond Laser Radiation under Ambient Condition. Nanoscale Research Letters, 4, 1263-1266. https://doi.org/10.1007/s11671-009-9390-y

[24] Reed, G.T., Mashanovich, G., Gardes, F.Y. and Thomson, D.J. (2010) Silicon Optical Modulators. Nature Photonics, 4, 518-526. https://doi.org/10.1038/nphoton.2010.179

[25] Ikushima, A.J., Fujiwara, T. and Saito, K. (2000) Silica Glass: A Material for Photonics. Journal of Applied Physics, 88, 1201-1213. https://doi.org/10.1063/1.373805

[26] Schweizer, T., Brady, D.J. and Hewak, D.W. (1997) Fabrication and Spectroscopy of Erbium Doped Gallium Lanthanum Sulphide Glass Fibres for Mid-Infrared Laser Applications. Optics Express, 1, 102-107. https://doi.org/10.1364/OE.1.000102

[27] Abernethy, J.A., Gawith, C.B.E., Eason, R.W. and Smith, P.G.R. (2002) Demonstration and Optical Characteristics of Electro-Optic Bragg Modulators in Periodically Poled Lithium Niobate in the Near-Infrared. Applied Physics Letters, 81, 25142516. https://doi.org/10.1063/1.1510964

[28] Weis, R.S. and Gaylord, T.K. (1985) Lithium Niobate: Summary of Physical Properties and Crystal Structure. Applied Physics A, 37, 191-203. https://doi.org/10.1007/BF00614817

[29] Cystran (2018) Silicon. https://www.crystran.co.uk/optical-materials/silicon-si

[30] Cystran (2018) Gallium Lanthanum Sulphide. https://www.crystran.co.uk/optical-materials/gallium-lanthanum-sulphide-gls

[31] Ashby, M.F. (2013) Chapter 15 Material Profiles. In: Ashby, M.F., Ed., Materials and the Environment, Second Edition, Butterworth-Heinemann, Boston, 459-595.

[32] Gooch \& Housego (2018) Lithium Niobate Wafers. 
https://goochandhousego.com/product-categories/ln-wafers/

[33] Mills, B., Heath, D.J., Grant-Jacob, J.A. and Eason, R.W. (2018) Predictive Capabilities for Laser Machining via a Neural Network. Optics Express, 26, 17245-17253. https://doi.org/10.1364/OE.26.017245 